Int. J. Odontostomat., 11(4):387-392, 2017.

\title{
Cáncer Bucomaxilofacial en Niños III: Tumores Malignos del Tejido Blando
}

\author{
Maxillofacial Cancer in Pediatric Patients III: Malignant Tumors in Soft Tissue
}

\author{
Alejandra Fernández ${ }^{1,2}$; Francisca Becerra3; Javiera Rehbein ${ }^{3}$; Sebastián Acuña \\ Carolina Somarriva ${ }^{4}$; Albano Cáceres $^{2} \&$ Alfredo Esguep ${ }^{5}$
}

FERNÁNDEZ, A.; BECERRA, F.; REHBEIN, J.; ACUÑA, S.; SOMARRIVA, C.; CÁCERES, A. \& ESGUEP, A. Cáncer bucomaxilofacial en niños III: Tumores malignos del tejido blando. Int. J. Odontostomat., 11(4):387-392, 2017.

RESUMEN: El grupo de neoplasias malignas de tejido blando de la región de cabeza y cuello en pacientes pediátricos está representado por carcinomas, sarcomas, melanomas y tumores de diferenciación incierta. La neoplasia más prevalente en la población pediátrica es el Rabdomiosarcoma, seguido por el carcinoma de células escamosas. Los rangos de presentación son muy amplios, siendo los grupos entre 2-6 años y 15-19 años los que presentan mayor incidencia. Se ha planteado que la etiología de estas neoplasias es incierta. El tratamiento de estas neoplasias es comúnmente de enfoque multimodal, combinando un procedimiento quirúrgico con quimioterapia y radioterapia. El pronóstico y sobrevida del paciente dependerán principalmente del momento en que se realice el diagnóstico de la lesión. Un diagnóstico y tratamiento temprano favorecen las posibilidades de sobrevida y el pronóstico del paciente. Este estudio corresponde a la 3ra parte de "Cáncer bucomaxilofacial en niños". Se hará referencia a los distintos tumores malignos del tejido blando en la población pediátrica en el territorio de cabeza y cuello, abarcando sus generalidades, etiología, epidemiología, tratamiento y pronóstico.

PALABRAS CLAVE: cáncer oral, niño, maxilares, mandíbula, mucosa bucal, neoplasias maxilomandibulares.

\section{INTRODUCCIÓN}

Entre los diferentes tipos de neoplasias que se desarrollan en la población pediátrica a nivel de cabeza y cuello, el grupo de las neoplasias malignas de tejido blando está representado por carcinomas, sarcomas, melanomas y tumores de diferenciación incierta. En la Tabla I se observa la clasificación de las neoplasias malignas de tejidos blandos basado en la OMS 2002 y 2005 (Barnes et al., 2005).

Es considerado que la presencia de carcinomas, a diferencia de los adultos, son poco frecuente en este grupo y en caso de presentarse, generalmente ocurre a nivel nasofaríngeo y se detecta más bien por metástasis en linfonodos cervicales (Santander, 2013).

El grupo de los sarcomas representa entre un 5$15 \%$ de las neoplasias malignas pediátricas, siendo el grupo de rabdomiosarcomas de cabeza y cuello el más común de estos (Divyambika et al., 2012). Según las estadísticas del programa de cáncer infantil en Chile (PINDA), la distribución porcentual de los sarcomas de partes blandas en la infancia corresponden al $6 \%$ de las neoplasias malignas pediátricas y se presentan con mayor frecuencia en pacientes de sexo masculino. Según el MINSAL (2011), el rango de presentación varía entre los primeros días de vida hasta los 19 años de edad, donde la mayor incidencia es entre los grupos de 2 a 6 años y de 15 a 19 años de edad. En general, la edad de presentación de los sarcomas de partes blandas pediátricos es entre los 5-10 años de edad. Según el MINSAL la sobrevida del paciente dependerá del tamaño del tumor. Si este es menor a $5 \mathrm{~cm}$, la sobrevida alcanza a un $81 \%$, por el contrario si es mayor a $5 \mathrm{~cm}$ la sobrevida disminuye drásticamente a un $16 \%$. Cabe destacar que si la detección es temprana, la probabilidad de mejoría y sobrevida es mayor (Vargas et al., 2011).

${ }^{1}$ Universidad Andrés Bello, Santiago, Chile.

${ }^{2}$ Centro de Investigaciones Biomédicas, Universidad de Los Andes, Santiago, Chile.

${ }^{3}$ Cirujano Dentista, Práctica Privada, Santiago, Chile.

${ }^{4}$ Universidad Andrés Bello, Santiago, Chile.

${ }^{5}$ Especialista en Patología Bucomaxilofacial, Santiago, Chile. 
Tabla I. Clasificación de las neoplasias malignas de tejidos blandos de la OMS, de presentación pediátrica (Barnes et al., 2005).
1. Tumores fibroblásticos y miofibroblásticos
- Fibrosarcoma Infantil
2. Tumores de musculatura lisa
- Leiomiosarcoma
3. Tumores de musculatura esquelética
- Rabdomiosarcoma
4. Tumores de la nasofaringe
- Carcinoma nasofaríngeo
5. Melanoma maligno de la mucosa
6. Tumores de diferenciación incierta
- Sarcoma Sinovial
- Sarcoma Alveolar de partes blandas

Los melanomas corresponden a un grupo menos frecuentes de neoplasias que los anteriores y se caracterizan por presentar un mal pronóstico (Warszawik-Hendzel et al., 2014). El Sarcoma alveolar de partes blandas, es extremadamente raro y es considerado dentro del grupo de tumores de diferenciación incierta. Esta neoplasia muestra predilección por la población pediátrica y adolescente, donde el sexo femenino es el más afectado (Fletcher et al., 2002).

Este estudio corresponde a la $3^{\text {ra }}$ parte de "Cáncer bucomaxilofacial en niños", donde se hará referencia a los distintos tumores malignos del tejido blando en la población pediátrica en el territorio de cabeza y cuello (tabla I), abarcando sus generalidades, etiología, epidemiología, tratamiento y pronóstico.

Tumores fibroblásticos y miofibroblásticos: Fibrosarcoma infantil. El fibrosarcoma infantil es histológicamente idéntico al fibrosarcoma de los adultos, pero presenta un pronóstico mucho más favorable. Se manifiesta en lactantes y niños, rara vez provoca metástasis y se manifiesta de forma similar a la fibromatosis (Fletcher et al.).

La OMS indica que representa aproximadamente un $13 \%$ de todos los tumores fibroblásticosmiofibroblásticos en niños y adolescentes y el $12 \%$ de los tumores malignos de tejidos blandos en lactantes. Un 36-80 \% de los casos son congénitos y un $36-100 \%$ de los casos ocurren durante el primer año de vida (Barnes et al.).

Según la distribución por sexo, existe controversia, por un lado Gosau et al. (2008), señalan que presenta igual distribución en hombres y mujeres, por el contrario, la OMS señala que es ligeramente mayor en el sexo masculino.

Con respecto al rango de edad más frecuente, la OMS señala que ocurre generalmente antes de los 3 años de edad (Barnes et al.) y Bang et al. (1989) postulan que varía entre el nacimiento y los 5 años de edad. La mayoría de los casos se ubican en las zonas más distales de las extremidades, por lo que la región de cabeza y cuello es escasamente afectada (Gosau et al.).

La OMS señala que su etiología es desconocida. No se ha demostrado la existencia de factores predisponentes específicos, ni enfermedades hereditarias o agentes causales relacionados.

El tratamiento de elección es la resección quirúrgica (Barnes et al.). El uso de radioterapia y quimioterapia se reserva para casos de alto grado histológico, que pueden presentarse con metástasis subclínicas y microscópicas al momento del diagnóstico (Gosau et al.).

El pronóstico es favorable y depende en gran medida del grado histológico del tumor y del éxito de la resección completa de la lesión (Gosau et al.). Aunque es una neoplasia de crecimiento rápido, las probabilidades de que genere metástasis son bajas, variando entre un 7,3-8,3\% de los casos en niños menores de 5 años. La tasa de recurrencia local es de 43 $\%$ y la de supervivencia es de 50-85 \% (Bang et al.).

Tumores de la musculatura lisa: Leiomiosarcoma: El leiomiosarcoma es una neoplasia maligna compuesta de células que exhiben características de músculo liso (Fletcher et al.). A nivel oral se sugiere que deriva de la túnica media de la pared vascular o de células pluripotenciales (Sandhu et al., 2014).

Representa entre el $5-10 \%$ de todos los sarcomas de tejidos blandos y en la región de cabeza y cuello menos del $10 \%$ de todos los leiomiosarcomas. Su presencia a nivel de la cavidad oral es menos común, siendo aún menos frecuente en la población pediátrica, lo que se puede atribuir a la escasa presencia de músculo liso en esta zona (Divyambika et al.).

Su etiología es desconocida, sin embargo se sugieren influencias hormonales debido a la predominancia del leiomiosarcoma retroperitoneal y de la vena cava inferior en mujeres (Fletcher et al.). 
El tratamiento consiste en primera instancia en la resección quirúrgica además de quimioterapia o radioterapia coadyuvante. La quimioterapia se utiliza con el objetivo de reducir las fallas en el tratamiento a largo plazo, mejorando la sobrevida y a su vez como tratamiento para evitar las metástasis. Se ha descrito que la radioterapia puede ser beneficiosa en lo que respecta a la disminución de la recurrencia local (Divyambika et al.).

Según la OMS los factores pronósticos más importantes son la ubicación y tamaño del tumor (Fletcher et al.). Adicionalmente, Divyambika et al., reconocen como indicadores de mal pronóstico aquellos casos que involucran adicionalmente tejido óseo y presentación de metástasis.

Tumores de la musculatura esquelética: Rabdomiosarcoma: Las variantes histológicas de esta neoplasia en niños incluyen al tipo embrionario y alveolar. El "Rabdomiosarcoma Embrionario" es un sarcoma primitivo de los tejidos blandos que presenta características biológicas y fenotípicas del músculo esquelético embrional. Esta neoplasia abarca los tipos de rabdomiosarcoma de células fusiforme, botrioide y anaplásico. El "rabdomiosarcoma alveolar" por su parte es una neoplasia maligna primitiva de células redondas que, citológicamente, asemeja a un linfoma y muestra diferenciación parcial de músculo esquelético (Fletcher et al.).

Es importante destacar que corresponde al grupo de sarcomas de tejidos blandos más común en la población pediátrica menor de 15 años de edad (Fletcher et al.). Este tumor representa aproximadamente un $60 \%$ de todos los sarcomas de partes blandas y aproximadamente un 3-8 \% de todos los cánceres que afectan al grupo pediátrico. La localización más frecuente es en la región de cabeza y cuello, donde la lengua, paladar, mejillas son los sitios más afectados (Chigurupati et al., 2002). Es más frecuente durante la primera década de la vida, expresando una mayor incidencia entre los 2-6 años de edad. Existe una leve predominancia en pacientes de sexo masculino y más común en niños caucásicos (Chigurupati et al.).

Su etiología es desconocida y la mayoría de los casos ocurren en forma esporádica sin ninguna predisposición ni factores de riesgos reconocibles (Sbeity et al., 2007). En el tipo embrional se han detectado la pérdida de heterocigosidad del cromosoma 11p15.5 y ganancias en los cromosomas 2, 8, 12 y 13. La región $11 \mathrm{p} 15.5$ contiene varios genes impresos, donde la pér- dida de un alelo podría inactivar el gen supresor de tumores o activar al protooncogen que condice al desarrollo del cáncer (Wang, 2012). En el tipo alveolar se observa la traslocación de $\mathrm{t}(2 ; 13)$ (q35; q14) o $\mathrm{t}$ $(1 ; 13)$ (p36; q14) (Manor et al., 2012).

Se plantea que su origen celular puede ser a partir de células miogénicas y a partir de células no musculares, es decir, a partir del tejido mesenquimal primitivo que expresa diferenciación miogénica, y probablemente surge de las células satélites asociadas con la embriogénesis del músculo esquelético (Wang).

El tratamiento consiste en una terapia multimodal, que incluye cirugía, radioterapia y quimioterapia. Los de tipo alveolar se tratan mediante quimioterapia adicional e iniciación de radioterapia. En los de tipo embrional el tratamiento incluye quimioterapia, sin aplicación de radioterapia (Hicks \& Flaitz, 2002).

El pronóstico para el tipo embrionario es más favorable que el de tipo alveolar, debido a que este último presenta un comportamiento más agresivo y metástasis. En gran medida, el tamaño y ubicación del tumor, estadiaje y edad del niño, influyen en el pronóstico de esta neoplasia (Wang).

En general, la tasa de supervivencia a los 5 años varía entre un $55-71 \%$ (Hicks \& Flaitz) y en los de tipo embrional alcanzan valores más altos, de hasta un 85 $\%$, pero estas tasas disminuyen según el avance del tumor al momento del diagnóstico (Wang).

Estos altos valores se deben a la aplicación de los protocolos de terapia multimodal sugeridos por el Intergroup Rhabdomyosarcoma Study Group (Wang), quienes establecieron cuatro etapas diferentes de progresión de esta lesión, donde según el avance y nivel de compromiso presente, se define el tratamiento a realizar. El objetivo de estos protocolos, es lograr que con el tratamiento quirúrgico definitivo se evite al máximo la utilización de quimioterapia o radioterapia, a fin de disminuir sus efectos adversos y complicaciones a largo plazo (Qaisi \& Eid, 2016). Actualmente, se espera que con este protocolo un $80 \%$ de los niños diagnosticados con esta neoplasia maligna se curen de su enfermedad (Hicks \& Flaitz).

Tumores de la nasofaringe: Carcinoma Nasofaríngeo: De los tumores nasofaríngeos el más frecuente es el carcinoma nasofaríngeo (Barnes et al.) y de tipo indiferenciado (Ozyar et al., 2006). La OMS lo define como un carcinoma que surge en la mucosa 
nasofaríngea y que muestra evidencia de diferenciación escamosa. Incluye al carcinoma de células escamosas, carcinoma no queratinizante y el carcinoma de células escamosas basaloide (Barnes et al.). Difiere a su contraparte en los adultos en su cercana asociación con la infección por virus de Epstein-Barr, en la alta incidencia del avance de la enfermedad al momento del diagnóstico (Polychronopoulou et al., 2004) y una mayor tasa de metástasis en los ganglios linfáticos (Ozyar et al.).

Representa aproximadamente el $1 \%$ de todas las neoplasias malignas en la población pediátrica a nivel mundial y es más frecuente en regiones del sur de Cina y sudeste asiático, con una incidencia intermedia en los países de la cuenca mediterránea, Alaska y Groenlandia (Ozyar et al.). Según Marcus \& Tishler (2010) esta neoplasia presenta predominancia por los niños de género masculino y la edad promedio al momento del diagnóstico es de 13 años de edad (Marcus \& Tishler).

El tratamiento consiste en radioterapia combinado con quimioterapia, pero tiene una mejor respuesta a la radioterapia. Esta modalidad combinada ha aumentado las tasas de supervivencia desde 20-60 $\%$ a $75-91 \%$ a los 5 años (Marcus \& Tishler). La sobrevida y pronósticos de los pacientes están directamente relacionados con la etapa de presentación, siendo el compromiso de linfonodos por metástasis un factor predictor de falla del tratamiento (Qaisi \& Eid).

Carcinoma de células escamosas: El Carcinoma de células escamosas es poco común en pacientes jóvenes y menos aún en la población pediátrica. Se plantea que etiológicamente es distinto al que se presenta en los adultos, ya que la exposición a factores de riesgo tales como el tabaco, alcohol o infecciones están normalmente ausentes en el grupo pediátrico. En algunos casos en los niños se ha asociado como factor contribuyente a la anemia de Fanconi o Xeroderma pigmentoso (Morris \& Ganly, 2010).

Aproximadamente uno de cada mil casos de carcinoma de células escamosas de cabeza y cuello ocurre en pacientes menores de 20 años de edad (Morris \& Ganly). A nivel oral, las ubicaciones más frecuentes afectadas en los niños son la lengua, los labios, encía y alveolos maxilares (Binahmed et al., 2007).

Por un lado se estima que esta neoplasia es más agresiva y de peor pronóstico que la contraparte en adulto (Binahmed et al.) y por otro el SEER estima que los pacientes pediátricos con carcinoma de células escamosas presentan mejores resultados de sobrevivencia que los adultos, por lo que se sugiere como tratamiento de elección la resección quirúrgica con márgenes de seguridad (Morris \& Ganly).

Melanoma de la mucosa: El melanoma es una neoplasia maligna derivada de los melanocitos o precursores melanocíticos. Se caracteriza por una proliferación de melanocitos atípicos en la interfase epitelioconectivo, con migración hacia el tejido epitelial e invasión al tejido conectivo subyacente (Barnes et al.). En la población pediátrica se clasifican en 4 categorías: melanomas que surgen en nevos gigantes congénitos; melanomas que surgen de otros nevos y de novo; melanomas del sistema nervioso central; y otras presentaciones (por ejemplo, transplacentaria) presentan un comportamiento menos agresivo comparado con los adultos, por lo que se sugiere que presentan un fenotipo diferente (Owens et al., 2002). El melanoma de la mucosa es poco frecuente en la región de cabeza y cuello, con aproximadamente 1000 casos reportados en la literatura (Owens et al.).

Aparentemente, los casos pediátricos de melanoma de la mucosa pueden ser apropiadamente tratados por medio de la escisión quirúrgica. El pronóstico de pacientes con melanoma de la mucosa en la región de cabeza y cuello es pobre (WarszawikHendzel et al.), donde la sobrevida media es de dos años, y a cinco años se estima que es menor a un 20 $\%$. Adicionalmente, las lesiones mayores a $5 \mathrm{~mm}$ de profundidad tienen significativamente un peor pronóstico (Fletcher et al.).

Tumores de diferenciación incierta: Sarcoma alveolar de partes blandas: El sarcoma alveolar de partes blandas es un tumor poco común que afecta principalmente a adolescentes y adultos jóvenes. Esta neoplasia representa aproximadamente el 0,4-1 \% de todos los casos de sarcomas de partes blandas y un 5 $\%$ de todos los sarcomas pediátricos de partes blandas. Durante las dos primeras décadas de vida, presenta cierta predilección por el género femenino y el sitio de origen más común es la región de cabeza y cuello, especialmente en la órbita y la lengua (Noussios et al., 2010).

El tumor se compone de células epiteliales largas y uniformes, con un abundante citoplasma granular eosinófilo, que se disponen en nidos sólidos y/o estructuras alveolares separadas por delgados vasos sinusoides (Fletcher et al.). Se plantea que tiene de- 
rivación miogénica o neuroendocrina, aunque ninguna de estas dos teorías ha sido probada. En algunos casos se ha encontrado asociado a su etiopatogenia alteraciones estructurales del cromosoma 17 que involucra a la banda q25 y traslocación del x;17 (Noussios et al.).

Según Noussios et al., sólo una docena de casos se han reportado en niños menores de 5 años en los últimos 50 años. Además, ellos exponen el caso de un niño de 3 años de edad, quien presentó esta neoplasia en el dorso lingual (Noussios et al.).

El tratamiento es principalmente quirúrgico, con márgenes de seguridad amplios. También se incluye como terapia coadyuvante la radioterapia y quimioterapia, pero su rol es limitado. El pronóstico se basa en la recurrencia local, las metástasis (Noussios et al.), la edad y el tamaño del tumor (Fletcher et al.), no obstante la recurrencia local es poco frecuente. En muchos casos las metástasis son la primera manifestación de la enfermedad, pudiendo hallarse en pulmones, huesos y cerebro (Noussios et al.).

CONCLUSIÓN: Ya expuestas las neoplasias malignas tanto de tejido duro como blando a nivel de la región de cabeza y cuello en el grupo pediátrico, podemos observar que claramente son más frecuentes a nivel del tejido óseo. Además, presentan diferencia en cuanto a los tipos más frecuentes en cada grupo. Así, en el tejido óseo las más representativas en orden decreciente son los Linfomas, el Osteosarcoma y la Histiocitosis de Langerhans. En cambio, en el tejido blando los más habituales en orden decreciente son el Rabdomiosarcoma y el Carcinoma de células escamosas. Podemos agregar que la etiopatogenia en este grupo de neoplasias malignas en el tejido blando también es controversial, como en el caso del grupo del tejido óseo.

Cabe destacar que de la información disponible en las bases de datos PubMed, CENTRAL y EBSCO, la mayoría de la evidencia es en base a reportes de casos y agregando a esto el escaso número registrados, hacen difícil evaluar la epidemiología, comportamiento y tratamiento de los diferentes tipos de neoplasia. Además, se desconoce si estos reportes de casos representan exactamente la historia natural de las neoplasias. Tampoco se conocen las secuelas a largo plazo asociadas a los tratamientos, sobre todo en lo que respecta al uso de radiación ionizante en niños.

Por estas razones, planteamos la necesidad de realizar un seguimiento de los pacientes durante un de- terminado periodo de tiempo, para poder evaluar realmente el efecto a largo plazo de las terapias coadyuvantes. Sin embargo, podemos concluir que un diagnóstico temprano favorece el pronóstico y que los diagnósticos tardíos, sumado a la poca diferenciación histológica, tamaño del tumor y presencia de metástasis, destinan significativamente a un peor pronóstico.

Recomendamos que el odontólogo se relacione con el cáncer fundamentalmente estableciendo prevención y diagnóstico temprano de las neoplasias. De esta manera, y al igual que en el adulto, todo niño debiese ser sometido a un control oral de rutina semestral o anual, que conste de exámenes clínicos y radiográficos de ser necesarios, con particular atención a partir de los 5 años, ya que se ha visto una mayor incidencia de lesiones malignas a partir de esta edad.

Frente a cualquier aumento de volumen y/o asimetría que aparezca durante la niñez, sin origen ni causa aparente, y por más inocua que la lesión parez$\mathrm{ca}$, es importante establecer una adecuada evaluación clínica, imagenológica e histológica antes de diagnosticarla como benigna o maligna, requisito esencial para determinar el manejo y tratamiento de cada paciente en particular.

FERNÁNDEZ, A.; BECERRA, F.; REHBEIN, J.; ACUÑA, S.; SOMARRIVA, C.; CÁCERES, A. \& ESGUEP, A. Maxillofacial cancer in pediatric patients III: Malignant tumors in soft tissue. Int. J. Odontostomat., 11(4):387-392, 2017.

ABSTRACT: Head and neck malignant tumors in pediatric patients comprise carcinoma, sarcoma, melanoma and tumours of uncertain differentiation. Within the pediatric population, the most prevalent neoplasm is rhabdomyosarcoma, followed by squamous cell carcinoma. There is a wide range in the presentation, and it varies significantly with age groups of 2-6 and 15-19 year-olds who present the higher incidence rates. For this reason, it has been suggested that the etiology of head and neck neoplasms remains unclear. Treating these pathologies usually involves a multimodal approach that combines surgery, radiation and chemotherapy. Prognosis and survival rates depend mainly of the stage at the time of diagnosis. Early diagnosis and treatment can improve prognosis and survival rates. In this 3rd part of "Maxillofacial Cancer in Pediatric Patients", we studied a variety of malignant tumors in head and neck soft tissue from a paediatric sample. Specifically, we aim to analyze their etiology, epidemiology, treatment and prognosis.

KEY WORDS: mouth neoplasms, child, maxillary, mandible, jaw neoplasms, tumors, mouth mucosa. 


\section{REFERENCIAS BIBLIOGRÁFICAS}

Bang, G.; Baardsen, R. \& Gilhuus-Moe, O. Infantile fibrosarcoma in the mandible: case report. J. Oral Pathol. Med., 18(6):339-43, 1989.

Barnes, L.; Eveson, J. W.; Reichart, P. \& Sidransky, D. (Eds.). World Health Organization Classification of Tumours. Pathology \& Genetics Head and Neck Tumours. Lyon, IARC Press, 2005.

Binahmed, A.; Charles, M.; Campisi, P.; Forte, V.; Carmichael, R. P. \& Sándor, G. K. Primary squamous cell carcinoma of the maxillary alveolus in a 10-year-old girl. J. Can. Dent. Assoc., 73(8):715-8, 2007.

Chigurupati, R.; Alfatooni, A.; Myall, R. W.; Hawkins, D. \& Oda, D. Orofacial rhabdomyosarcoma in neonates and young children: a review of literature and management of four cases. Oral Oncol., 38(5):508-15, 2002.

Divyambika, C. V.; Sathasivasubramanian, S.; Krithika, C. L.; Malathi, N. \& Prathiba, D. Pediatric oral leiomyosarcoma: rare case report. J. Cancer Res. Ther., 8(2):282-5, 2012.

Fletcher, C. D. M.; Unni, K. K. \& Mertens, F. (Eds.). World Health Organization Classification of Tumours Pathology and Genetics of Tumours of Soft Tissue and Bone. Lyon, IARC Press, 2002.

Gosau, M.; Draenert, F. G.; Winter, W. A.; Mueller-Hoecker, J. \& Driemel, O. Fibrosarcoma of the childhood mandible. Head Face Med., 4:21, 2008.

Hicks, J. \& Flaitz, C. Rhabdomyosarcoma of the head and neck in children. Oral Oncol., 38(5):450-9, 2002.

Manor, E.; Joshua, B. Z.; Nash, M.; Brennan, P. A. \& Bodner, L. Cytogenetics of primary embryonal rhabdomyosarcoma of the cheek. Br. J. Oral Maxillofac. Surg., 50(8):788-90, 2012.

Marcus, K. J. \& Tishler, R. B. Head and neck carcinomas across the age spectrum: epidemiology, therapy, and late effects. Semin. Radiat. Oncol., 20(1):52-7, 2010.

Morris, L. G. \& Ganly, I. Outcomes of oral cavity squamous cell carcinoma in pediatric patients. Oral Oncol., 46(4):292-6, 2010.

Noussios, G.; Chouridis, P.; Petropoulos, I.; Karagiannidis, K. \& Kontzoglou, G. Alveolar soft part sarcoma of the tongue in a 3-year-old boy: a case report. J. Med. Case Rep., 4:130, 2010.

Owens, J. M.; Gomez, J. A. \& Byers, R. M. Malignant melanoma in the palate of a 3-month-old child. Head Neck, 24(1):91-4, 2002.

Ozyar, E.; Selek, U.; Laskar, S.; Uzel, O.; Anacak, Y.; BenArush, M.; Polychronopoulou, S.; Akman, F.; Wolden, S. L.; Sarihan, S.; Miller, R. C.; Ozsahin, M.; Abacioglu, U.; Martin, M.; Caloglu, M.; Scandolaro, L.; Szutowicz, E. \& Atahan, I. L. Treatment results of 165 pediatric patients with non-metastatic nasopharyngeal carcinoma: a Rare Cancer Network study. Radiother. Oncol., 81(1):39-46, 2006.

Polychronopoulou, S.; Kostaridou, S.; Panagiotou, J. P.; Stefanaki, K.; Papadakis, V.; Florentin, L.; Houlakis, M.;
Christopoulos, G. \& Haidas, S. Nasopharyngeal carcinoma in childhood and adolescence: a single institution's experience with treatment modalities during the last 15 years. Pediatr. Hematol. Oncol., 21(5):393-402, 2004.

Qaisi, M. \& Eid, I. Pediatric head and neck malignancies. Oral Maxillofac. Surg. Clin. North Am., 28(1):11-9, 2016.

Sandhu, S. V.; Sodhi, S. P.; Rai, S. \& Bansal, H. Primary leiomyosarcoma of the maxilla: An investigative loomreport of a challenging case and review of literature. J. Oral Maxillofac. Pathol., 18(3):453-9, 2014.

Santander, S. División Prevención y Control de Enfermedades, Departamento de Enfermedades No Transmisibles. Santiago de Chile, Ministerio de Salud, 2013.

Sbeity, S.; Abella, A.; Arcand, P.; Quintal, M. C. \& Saliba, I. Temporal bone rhabdomyosarcoma in children. Int. J. Pediatr. Otorhinolaryngol., 71(5):807-14, 2007.

Vargas, L.; Varas, M.; Campbell, M. \& Salgado, C. Cuando Sospechar un Cancer en el Niño y como Derivar. Santiago de Chile, Subsecretaria de Salud Publica, Ministerio de Salud, Gobierno de Chile, 2011.

Wang, C. Childhood rhabdomyosarcoma: recent advances and prospective views. J. Dent. Res. 91(4):341-50, 2012.

Warszawik-Hendzel, O.; S?owin'ska, M.; Olszewska, M. \& Rudnicka, L. Melanoma of the oral cavity: pathogenesis, dermoscopy, clinical features, staging and management. J. Dermatol. Case Rep., 8(3):60-6, 2014.

Dirección para correspondecia

Alejandra Fernández Moraga

Universidad Andrés Bello

Echaurren 237

Santiago

CHILE

Email: fernandez.alejandra@gmail.com

Recibido : 06-05-2017

Aceptado: 21-09-2017 